\title{
Predictors of adherence to sublingual immunotherapy for Japanese cedar pollinosis: a prospective analysis
}

\author{
Sayaka Kikkawa, ${ }^{1,3,5}$ Atsushi Kamijo, ${ }^{1,3}$ Kazuyuki Nakagome, ${ }^{2,3}$ Tomoyuki Soma, ${ }^{2,3}$ Takehito Kobayashi, ${ }^{3,4}$ \\ Yoshitaka Uchida, ${ }^{2,3}$ Yasuhiro Kase, ${ }^{1,3}$ Makoto Nagata ${ }^{2,3}$
}

\begin{abstract}
Background: Sublingual immunotherapy (SLIT) often has low adherence rates.

Objective: To provide effective support for SLIT continuation, we investigated potential predictors of SLIT adherence through a prospective analysis of patient characteristics. We excluded evaluation of treatment effect and symptoms during treatment, aiming instead to identify predictors of later dropout or insufficient adherence due to indolence or forgetfulness using only information obtained at initial examination.
\end{abstract}

Methods: We provided patients with a questionnaire and monitored self-reported adherence once every 6 months. Cases of dropout for clear reasons were excluded, but cases of dropout or insufficient adherence to SLIT for indolence or forgetfulness were included.

Results: Fifty-three patients receiving SLIT were assessed. Nine patients dropped out after providing a clear reason. Thirty-four patients maintained good adherence. Seven patients continued SLIT but with insufficient adherence, while three patients discontinued SLIT for unclear reasons (indolence or forgetfulness) and these ten individuals were classified as the poor-adherence group. Univariate analysis and multivariate logistic regression analysis of the good-adherence and poor-adherence groups showed age to be a significant predictor of SLIT adherence. Based on analysis of a receiver operating characteristic curve, age $<40.5$ years was selected as the optimal cutoff value for predicting poor adherence to SLIT.

Conclusion: To prevent treatment SLIT discontinuation on account of indolence or forgetfulness, the necessity of longterm treatment continuity should be communicated clearly prior to commencing treatment, especially for patients under 40 years of age.

Key words: Adherence, Japanese cedar pollinosis, patient characteristics, questionnaire, sublingual immunotherapy

\footnotetext{
Affiliations:

${ }^{1}$ Department of Otorhinolaryngology,

${ }^{2}$ Department of Respiratory Medicine,

3 Allergy Center,

${ }^{4}$ Department of General Internal Medicine,

Saitama Medical University Hospital, Saitama, Japan

Department of Otolaryngology, Chigasaki Central Hospital, Kanagawa, Japan
}

Corresponding author:

Sayaka Kikkawa

Department of Otorhinolaryngology, Saitama Medical

University Hospital,

38 Morohongo, Moroyama-machi, Iruma-gun, Saitama,

350-0495, Japan

E-mail: skikkawa@saitama-med.ac.jp

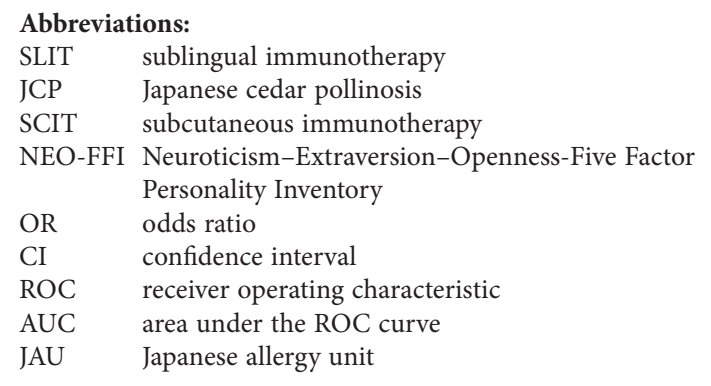

\section{Introduction}

An extract for sublingual immunotherapy (SLIT) for Japanese cedar pollinosis (JCP) became available commercially in Japan in 2014. The clinical efficacy of allergen immunotherapy requires administration of standardized allergen extracts in adequate doses and for sufficient periods of time (3-5 years). 
Low adherence rates represent a considerable problem for the success of long-term treatments.

SLIT is self-administered by patients without medical supervision. Home administration removes one of the major obstacles to adherence because it does not require the patient to attend a physician's office for treatment, unlike subcutaneous immunotherapy (SCIT). However, SLIT adherence varies widely, with dropout rates ranging from $49 \%$ to $82 \%$ of patients prescribed three years of SLIT. ${ }^{1,2,3}$ Therefore, if clinicians could predict SLIT adherence to medication prior to commencing treatment: (i) correct guidance to patients can be given; and (ii) appropriate patients can be selected for SLIT.

To provide effective support for continued treatment, we investigated potential predictors of SLIT adherence through a prospective analysis of patient characteristics.

Unlike previous reports, our study did not simply analyze reasons for dropout. Instead, we attempted to predict dropout or insufficient adherence due to indolence or forgetfulness using information available prior to commencing treatment. Therefore, we excluded evaluation of treatment effect and symptoms during treatment, aiming to identify predictors using only information obtained at initial examination.

\section{Methods}

\section{Participants and study procedure}

This was a prospective observational study registered in the UMIN clinical trial registry website (UMIN000015652). We recruited 53 adults (all aged $>20$ years) who started SLIT for JCP from November 2014 to August 2016 at Saitama Medical University Hospital. The study was carried out over more than two pollen seasons.

\section{Ethics statement}

The study protocol was approved by the Institutional Review Board of Saitama Medical University Hospital (IRB No.14086). Written informed consent was obtained from all patients.

\section{SLIT protocol}

Patients received a standardized JCP extract (Cedartolen ${ }^{\circ}$ Sublingual Drops; Torii Pharmaceuticals, Tokyo, Japan). In the SLIT protocol for $\mathrm{JCP}^{4}$ the patient receives escalating doses for 2 weeks, beginning with $0.2 \mathrm{~mL}$ of JCP extract [200 Japanese allergy unit (JAU)/mL] and increasing by $0.2 \mathrm{~mL}$ every second day up to $1.0 \mathrm{~mL}$ at $200 \mathrm{JAU} / \mathrm{mL}$. Thereafter, treatment is switched to $0.2 \mathrm{~mL}$ of JCP extract $(2000 \mathrm{JAU} / \mathrm{mL})$ and increases similarly until reaching the maintenance dose of 1.0 $\mathrm{mL}(2000 \mathrm{JAU} / \mathrm{mL})$. Starting from the third week, patients receive a daily maintenance dose of $1.0 \mathrm{~mL}$ of JCP extract at $2000 \mathrm{JAU} / \mathrm{mL}$.

The SLIT protocol has been described previously. Patients attempted to take the medication for the first time after a description of SLIT had been given by a physician or nurse. After ascertaining if early side effects (30 min) had occurred, questionnaires were handed out to patients. For patients who discontinued treatment, we asked them their reasons for doing so by telephone.

\section{Questionnaire}

Questions on patient characteristics

Questions on patient characteristics were divided broadly into three sections:

1) Sexuality, age, age at disease onset, disease duration.

2) Time required to visit the hospital; distance to the hospital; ease of visiting the hospital; regularity of sleep schedule and waking up at a certain time; regularity of eating breakfast; child care or family care; hobbies; alcohol consumption; tobacco smoking; marital status; highest level of education attained; annual income; profession; medical history; and current medication use.

3) JCP type (sneeze and rhinorrhea/nasal blockage/combined type) and severity (most severe/severe/moderate/ mild) as measured via the Total Nasal Symptom Score.

\section{Questions on level of understanding of treatment}

In this section, patients were asked to answer ten questions with "yes", "no" or "don't know":

Q1: SLIT should be taken every day.

Q2: SLIT has no side effects.

Q3: SLIT must be stopped when receiving dental treatment or if mouth ulcers develop.

Q4: SLIT can be resumed immediately even after a washout period of $>1$ month.

Q5: Alcohol can be consumed immediately after SLIT.

Q6: Exercise should not be performed immediately before or after SLIT.

Q7: SLIT can resolve mite allergy as well as JCP.

Q8: SLIT can resolve JCP completely in all patients.

Q9: SLIT can be used in combination with other medications during JCP season.

Q10: SLIT is complete after 1 year.

\section{Questions on personality traits}

We assessed personality traits using the NeuroticismExtraversion-Openness-Five Factor Personality Inventory (NEO-FFI). ${ }^{5}$ The inventory consists of 60 items, including 12 items for each of the five domains (neuroticism, extraversion, openness, agreeableness and conscientiousness).

\section{Questions on medication adherence}

We monitored self-reported adherence to SLIT once every 6 months. Patients answered questions regarding the previous 6 months by stating how often they took their medication:

Q1: Every day.

Q2: Almost every day.

Q3: 5 days/week.

Q4: About 3-4 days/week

Q5: Only 1-2 days/week

Q6: Sporadically.

Q7: Treatment discontinued. 


\section{Definitions}

"Dropout" was defined as stopping treatment and never resuming it. "Insufficient adherence" was defined as self-administration of SLIT at a frequency of any of: 5 days/week, about 3-4 days/week, only 1-2 days/week, or sporadically. "Sufficient adherence" was defined as self-administration of SLIT every day or almost every day.

\section{Statistical analyses}

Quantitative variables were expressed as means \pm standard deviations. Normality of the data was verified using the Shapiro-Wilk test. The Mann-Whitney U-test and unpaired Student's t-test were used to assess differences between two groups. Pearson's chi-squared test or Fisher's exact test were used to assess associations between two categorical variables. Multivariable logistic regression analysis was used to evaluate the combined effects of risk factors. Variables with $p<0.10$ in univariate analysis were included in the multivariable logistic regression model-building process; $p<0.05$ was considered statistically significant. Odds ratios (ORs) are presented with 95\% confidence intervals (CIs). All statistical analyses were performed using SPSS v24.0 (IBM, Armonk, NY, USA).

\section{Results}

Fifty-three patients (25 males and 28 females; mean age, $48.0 \pm 14.3$ years) formed the study cohort. Nine patients dropped out because of a definite reason (e.g., early side effects, moving out of the area, or no effect of SLIT). The patients who moved out of the area were forced to change their living location relatively early after treatment initiation. Therefore, it was not possible to periodically confirm adherence thereafter, and it is unclear whether treatment adherence was sufficient. We think that it is necessary to exclude these individuals at this time. The single patient reporting no effect of SLIT was an 82-year-old man. At first we thought that he should be excluded from treatment because of his advanced age, but he was very hopeful that SLIT might be of help. However, he complained that his nasal discharge remained regardless of SLIT. We assumed that his nasal discharge was not solely due to cedar pollen, and might have other physiological explanations related to advanced age. Within a year, this patient dropped out after losing interest in SLIT and we therefore excluded him from our analyses.

Thirty-four patients maintained good adherence to SLIT, and we classified them as the "good-adherence group". Seven patients continued SLIT despite insufficient adherence and three cases discontinued SLIT for unclear reasons (indolence or forgetfulness). Together, these ten patients formed the "poor-adherence group". We then made comparisons between the good-adherence group $(\mathrm{n}=34)$ and the poor-adherence group $(\mathrm{n}=10)$.

\section{Patient characteristics}

We analyzed the 12 items which received appropriate responses in our questionnaire (sex; age; age at disease onset; disease duration; ease of visiting the hospital; regularity of sleep schedule and waking up at a certain time; regularity of breakfast consumption; child care or family care; alcohol consumption; tobacco smoking; JCP type; JCP severity).

Univariate analysis of patients in the good-adherence and poor-adherence groups showed that age was associated with SLIT adherence (Table 1). Multivariate logistic regression analysis using the three factors attaining $p<0.1$ in univariate analyses revealed that age (OR $0.89,95 \%$ CI $0.81-0.97 ; p$ $=0.011$ ) remained a significant predictor of SLIT adherence. Based on analyses of a receiver operating characteristic (ROC) curve, age $<40.5$ years was selected as the optimal cutoff value for predicting poor adherence to SLIT. The sensitivity and specificity were $60.0 \%$ and $82.4 \%$, respectively, with an overall area under the ROC curve (AUC) of 0.766 (Figure 1).

Table 1. Characteristics of the study cohort (44 patients)

\begin{tabular}{|lccc|}
\hline & $\begin{array}{c}\text { Good adherence } \\
\mathbf{n}=\mathbf{3 4}\end{array}$ & $\begin{array}{c}\text { Poor adherence } \\
\mathbf{n}=\mathbf{1 0}\end{array}$ & $\boldsymbol{p}$ \\
\hline Male, number (\%) & $15(44.1)$ & $3(30.0)$ & 0.34 \\
\hline Age (years), mean \pm SD & $51.4 \pm 11.4$ & $39.8 \pm 11.3$ & 0.01 \\
\hline Age at JCP onset (years), mean \pm SD & $24.8 \pm 10.4$ & $21.4 \pm 9.4$ & 0.39 \\
\hline Duration of JCP (years), mean \pm SD & $26.3 \pm 11.4$ & $19.9 \pm 12.5$ & 0.15 \\
\hline Ease of visiting hospital, number (\%) & $14(43.8)$ & $7(77.8)$ & 0.06 \\
\hline not difficult & $15(46.9)$ & $1(11.1)$ & 0.59 \\
\hline slightly difficult & $3(9.4)$ & $1(11.1)$ & 0.42 \\
\hline very difficult & $28(82.4)$ & $8(80.0)$ & 0.26 \\
\hline Regular sleep schedule, number (\%) & $30(88.2)$ & $8(80.0)$ & 0.30 \\
\hline Regular breakfast consumption, number (\%) & $8(23.5)$ & $6(40.0)$ & \\
\hline Children or family care, number (\%) & $14(43.8)$ & & \\
\hline Alcohol consumption, number (\%) & & & \\
\hline
\end{tabular}


Table 1. (Continued)

\begin{tabular}{|c|c|c|c|}
\hline & $\begin{array}{l}\text { Good adherence } \\
\qquad \mathbf{n}=\mathbf{3 4}\end{array}$ & $\begin{array}{l}\text { Poor adherence } \\
\quad \mathrm{n}=10\end{array}$ & $p$ \\
\hline Tobacco smoking, number (\%) & $1(3.1)$ & $1(10.0)$ & 0.42 \\
\hline Type of JCP, number (\%) & & & 0.07 \\
\hline sneezing and rhinorrhea & $9(26.5)$ & $0(0.0)$ & \\
\hline nasal blockage & $5(14.7)$ & $3(30.0)$ & \\
\hline combined type & $20(58.8)$ & $7(70.0)$ & \\
\hline Severity of JCP, number (\%) & & & 0.35 \\
\hline mild & $3(8.8)$ & $2(20.0)$ & \\
\hline moderate & $5(14.7)$ & $1(10.0)$ & \\
\hline very severe or severe & $26(76.5)$ & $7(70.0)$ & \\
\hline
\end{tabular}

JCP: Japanese cedar pollinosis

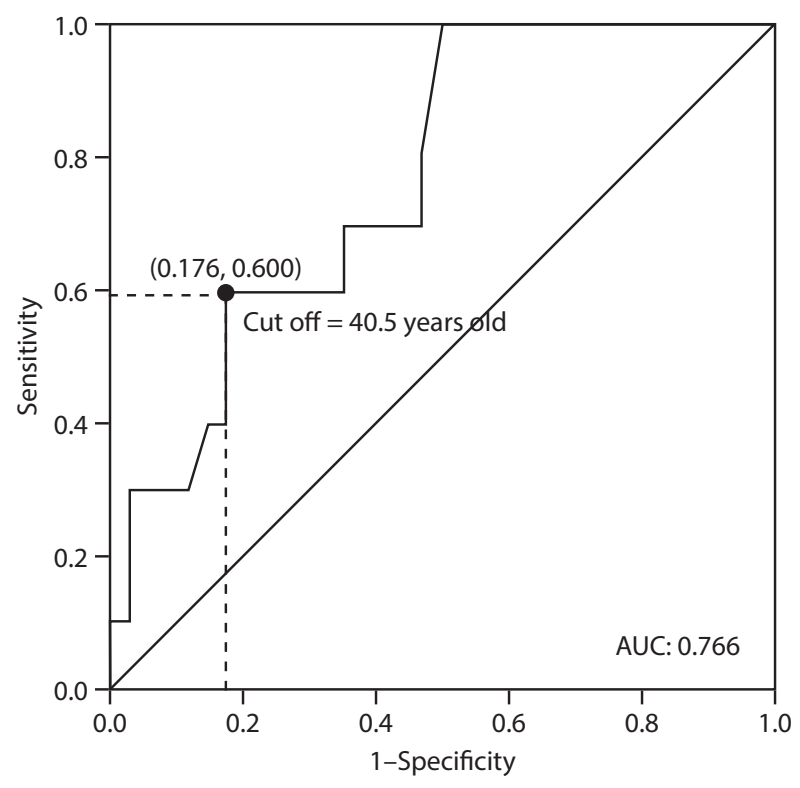

Figure 1. Analysis of receiver operating characteristic (ROC) curve for age as a predictor of SLIT adherence.

Age $<40.5$ years was selected as the optimal cutoff value for predicting poor adherence to SLIT. The sensitivity and specificity were $60.0 \%$ and $82.4 \%$, respectively, with an overall area under the ROC curve (AUC) of 0.766 .

\section{Level of understanding of treatment}

The mean number of correct answers to the questionnaire was $8.3 \pm 1.5$ in the good-adherence group and $8.1 \pm 2.4$ in the poor-adherence group. This difference was not statistically significant $(p=0.67)$.

\section{Personality traits}

There was no significant difference in five-dimensional NEO -FFI scores between the good-adherence and poor-adherence groups (Table 2 ).
Table 2. NEO-FFI scores (mean \pm SD) of study participants

\begin{tabular}{lccc} 
& $\begin{array}{c}\text { Good adherence } \\
\mathbf{n}=\mathbf{3 4}\end{array}$ & $\begin{array}{c}\text { Poor adherence } \\
\mathbf{n}=\mathbf{1 0}\end{array}$ & $\boldsymbol{p}$ \\
\hline Neuroticism & $52.9 \pm 19.5$ & $49.3 \pm 14.7$ & 0.6 \\
Extraversion & $52.2 \pm 11.6$ & $47.3 \pm 10.3$ & 0.24 \\
Openness & $53.0 \pm 8.5$ & $51.9 \pm 10.2$ & 0.73 \\
Agreeableness & $49.5 \pm 10.4$ & $54.4 \pm 8.4$ & 0.19 \\
\hline Conscientiousness & $50.3 \pm 8.3$ & $47.7 \pm 7.2$ & 0.37 \\
\hline
\end{tabular}

NEO-FFI, Neuroticism-Extraversion-Openness-Five Factor Personality Inventory

\section{Discussion}

Adherence to medication is crucial for all types of longterm treatments. SLIT is convenient for patients because treatment is self-administered and safe, ${ }^{6}$ but serious challenges regarding adherence remain.

In a 2011 Italian study, ${ }^{2}>50 \%$ of patients discontinued SLIT during the first year; by the second year, only $28 \%$ remained on treatment, and this figure declined to $13 \%$ in the third year. In a German study of SLIT for pollinosis, ${ }^{7} 47 \%$ of patients discontinued SLIT during the first year; by the second year, only $26 \%$ remained on treatment, and this figure declined to $16 \%$ in the third year. In a study in the Netherlands, ${ }^{8}$ only $7 \%$ of patients remained on SLIT by the third year of treatment.

In Japan, an extract for SLIT for JCP became available commercially in 2014. Most patients visited our hospital with the hope of receiving treatment around the same time as SLIT became available for JCP in Japan. Consistently, their symptoms were not sufficiently alleviated by drug treatment alone, so they were enthusiastic to receive SLIT for JCP. However, before starting SLIT, we always made sure to carefully explain SCIT and SLIT. Patients started treatment after having carefully considered which treatment they wished to receive. Several years have passed since SLIT for JCP was started to be used in clinical practice, and it is beginning to appear that 
treatment continuation rates are very high in Japan. Yuta and colleagues $^{9}$ reported on 133 patients undergoing SLIT for JCP. They found that $3.1 \%$ of patients discontinued treatment during the first year, and $4.1 \%$ did so in the second year.

Moreover, in 2016, Yuta and co-workers ${ }^{10}$ reported that treatment outcomes using a visual analog scale (VAS), a face scale, and the Total Nasal Symptom Score were improved significantly in patients whose SLIT adherence was over $70 \%$ or $75 \%$. An adherence rate of $70 \%$ is equivalent to taking medication 5 days/week, so instructing patients not to forget their medication more than once or twice per week is important. However, Yuta and colleagues also pointed out that adherence rates reported using a questionnaire were $13.5 \%$ higher than those measured using prescription monitoring, so the limitations of our study include the method used to investigate adherence (a questionnaire).

Another limitation of our study was the loss to follow up of patients who moved out of the area, which may have introduced selection bias into the results.

A further limitation of our study was that the number of cases was quite small. The reason for the small sample size is that this was a prospective study at a single institution, and SLIT for JCP has been used in Japan for only a short period starting in 2014. Only 33 cases have been followed over time in other single-institution studies ${ }^{11}$ in Japan, and even in multi-institutional research, ${ }^{12}$ the sample size is 356 cases in total. Compared with other countries, Japanese patients have very low dropout rates from SLIT. Therefore, the number of individuals with poor adherence to SLIT studied here was unavoidably quite small.

In conducting this study, we considerably discussed the definition of adherence. Considering the high continuation rates in Japan and the purpose of our study, we needed to distinguish patients who dropped out for clear reasons and those who dropped out because of indolence or forgetfulness. Although there have been some retrospective investigations of dropout reasons in previous Japanese and international reports, our prospective study examined whether it was possible to predict adherence from information obtained at the patient's initial visit. We believe that this represents a novel hypothesis. Our conclusions were that relatively young patients under the age of 40.5 years tended to have inadequate adherence due to indolence or forgetfulness. Other studies investigating adherence to medications for chronic diseases (including immunotherapy) have reported differences in treatment adherence associated with patient age.,13-16 In reports from Japan, age and occupation have both been reported to be strongly associated with low adherence to treatment. ${ }^{16}$ Moreover, Hashimoto and colleagues reported that adherence rates were lower in employed persons. ${ }^{17}$ Even though these patients may be willing to start treatment, it may not be continued, perhaps because younger individuals are often busy with labor, child care and other responsibilities. With this in mind, we believe that more efficient SLIT treatment can be achieved.

\section{Conclusions}

To prevent treatment SLIT discontinuation on account of indolence or forgetfulness, the necessity of long-term treatment continuity should be communicated clearly prior to commencing treatment, especially for patients under 40 years of age.

\section{Acknowledgements}

We thank Arshad Makhdum, PhD, from Edanz Group (www.edanzediting.com/ac) for editing a draft of this manuscript. MN received honoraria from Astra Zeneca, GSK, Novartis Pharma and Torii Pharmaceuticals.

\section{Trial registry}

This trial was registered in the UMIN clinical trial registry (UMIN000015652) on November 10, 2014.

\section{References}

1. Bender BG, Oppenheimer J. The special challenge of nonadherence with sublingual immunotherapy. J Allergy Clin Immunol Pract. 2014;2:152-5.

2. Senna G, Lombardi C, Canonica GW, Passalacqua G. How adherent to sublingual immunotherapy prescriptions are patients? The manufacturers' viewpoint. J Allergy Clin Immunol. 2010;126:668-9.

3. Sieber J, De Geest S, Shah-Hosseini K, Mosges R. Medication persistence with long-term, specific grass pollen immunotherapy measured by prescription renewal rates. Curr Med Res Opin. 2011;27:855-61.

4. Yamanaka K, Shah SA, Sakaida H, Yamagiwa A, Masuda S, Mizutani H, et al. Immunological parameters in prophylactic sublingual immunotherapy in asymptomatic subjects sensitized to Japanese cedar pollen. Allergol Int. 2015;64:54-9.

5. Costa PT Jr, McCrae RR. Revised NEO Personality Inventory (NEO-PI-R) and NEO Five-Factor Inventory (NEO-FFI) professional manual. Odessa (FL): Psychological Assessment Resources; 1992.

6. Aboshady OA, Elghanam KM. Sublingual immunotherapy in allergic rhinitis: efficacy, safety, adherence and guidelines. Clin Exp Otorhinolaryngol. 2014;7:241-9.

7. Egert-Schmidt AM, Kolbe JM, Mussler S, Thum-Oltmer S Patients' compliance with different administration routes for allergen immunotherapy in Germany. Patient Prefer Adherence. 2014;8:1475-81.

8. Kiel MA, Roder E, van Gerth WR, Al MJ, Hop WC, Rutten-van Molken MP. Real-life compliance and persistence among users of subcutaneous and sublingual allergen immunotherapy. J Allergy Clin Immunol. 2013; 132:353-60.

9. Yuta A, Ogawa Y, Suzuki Y, Arikata M, Kozaki H, Shimizu T, et al. [Clinical efficacy of sublingual immunotherapy in the second treated year for 133 patients with Japanese cedar pollinosis in 2016]. Arerugi. 2016;65:1209-18. Japanese.

10. Yuta A, Ogawa Y, Suzuki Y, Arikata M, Kozaki H, Shimizu T, et al. [Adherence and clinical outcome of sublingual immunotherapy for Japanese cedar pollinosis]. Nippon Jibiinkoka Gakkai Kaiho. 2016;119: 1504-10. Japanese.

11. Onishi K, Kawashima K, Yamato A, Yasui T. [Investigation of sublingual Immunotherapy for patients with cedar pollinosis]. Nihon Bika Gakkai Kaishi. 2018;57:572-80. Japanese.

12. Ohta N, Yuta A, Ogawa Y, Shoji F, Awataguchi T, Suzuki N, et al. [Practical study of sublingual immunotherapy in patients with Japanese cedar pollinosis using a questionnaire comparison among the first season, second season and third season]. Nippon Jibiinkoka Gakkai Kaiho. 2019; 122:35-44. Japanese.

13. Lemberg ML, Berk T, Shah-Hosseini K, Kasche EM, Mosges R. Sublingual versus subcutaneous immunotherapy: patient adherence at a large German allergy center. Patient Prefer Adherence. 2017;11:63-70.

14. Bandi P, Goldmann E, Parikh NS, Farsi P, Boden-Albala B. Age-related differences in antihypertensive medication adherence in Hispanics: a cross-sectional community-based survey in New York City, 2011-2012. Prev Chronic Dis. 2017;14:E57. 
15. Toyosawa E, Mieno E, Tsutsumi K, Nakamura K, Nakano S, Eshima N. Analysis of factors related to medication compliance in elderly patients: a survey of outpatients in the department of internal medicine of Oita medical university hospital. Jpn J Clin Pharmacol Ther. 1997;28:667-81.
16. Osanai Y, Katsura S, Sato H, Kimura R, Kodama H, Takasugi K, et al. Evaluation of the factors influencing medicine-taking behavior for the patients taking oral medication. Jpn J Soc Pharm. 2015;34:72-80.

17. Hashimoto H, Tanaka M, Oyake T, Gomi T, Ikeda T, Yoshida M, et al. Study on factors that contribute to poor patient compliance with medication in Japan. Jpn J Hosp Pharm. 1999;25:634-42. 